\title{
Rayleigh Scattering Measurements Using a Tunable Liquid Crystal Fabry-Perot Interferometer
}

\author{
Amy F. Mielke-Fagan* \\ NASA Glenn Research Center, Cleveland, Ohio, 44135 \\ Michelle M. Clem ${ }^{\dagger}$ \\ NASA Glenn Research Center, Cleveland, Ohio, 44135 \\ Kristie A. Elam ${ }^{\ddagger}$ \\ Jacobs Technology, Cleveland, $\mathrm{OH} 44135$
}

\begin{abstract}
[Abstract] Spectroscopic Rayleigh scattering is an established flow diagnostic that has the ability to provide simultaneous density, velocity, and temperature measurements. The Fabry-Perot interferometer or etalon is a commonly employed instrument for resolving the spectrum of molecular Rayleigh scattered light for the purpose of evaluating these flow properties. This paper investigates the use of a tunable liquid crystal (LC) Fabry-Perot etalon in Rayleigh scattering experiments at NASA Glenn Research Center. The LC etalon provides a robust interferometry system that can be tuned rapidly by adjusting the voltage applied to the liquid crystal interface. Tuning the interferometer is often necessary to control the physical locations of the concentric interference fringes when Rayleigh light is imaged through the LC etalon. The LC etalon diagnostic system was tested in a $1-\mathrm{cm}$ diameter nozzle flow in two different scattering configurations to evaluate its usefulness for Rayleigh measurements compared to a traditional non-tunable fused silica Fabry-Perot etalon.
\end{abstract}

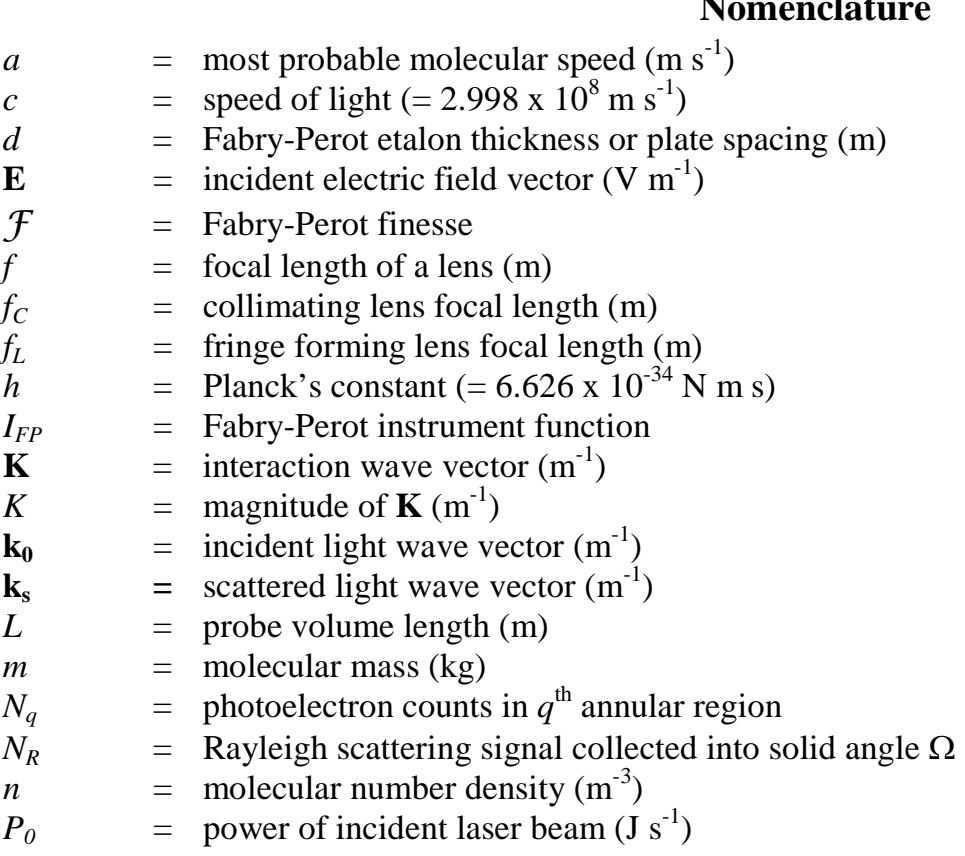

\footnotetext{
${ }^{*}$ Research Engineer, Optical Instrumentation and NDE Branch, 21000 Brookpark Road, AIAA Senior Member

${ }^{\dagger}$ Research Engineer, Optical Instrumentation and NDE Branch, 21000 Brookpark Road

${ }^{\ddagger}$ Optics Technician, 21000 Brookpark Road
} 


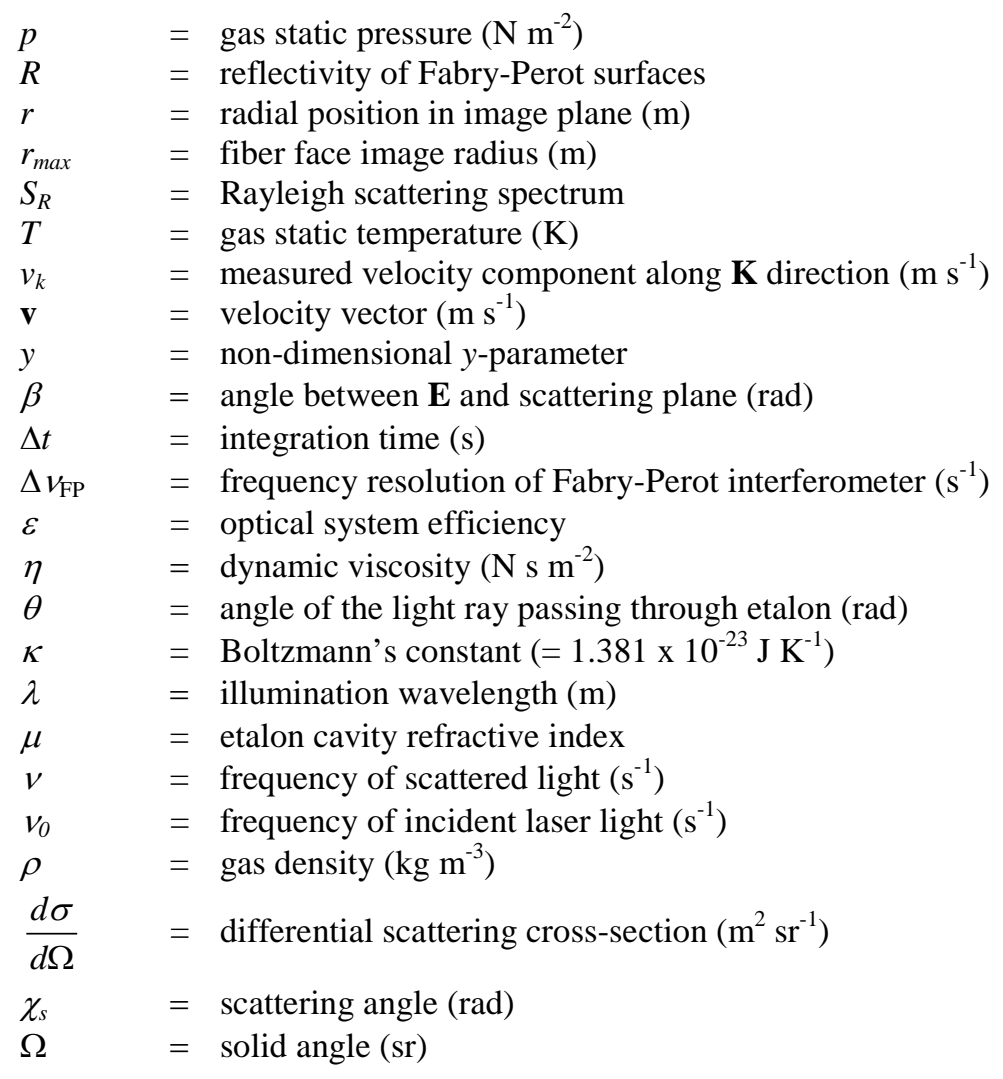

\section{Introduction}

R AYLEIGH scattering is the elastic scattering of light from molecules where the signal strength, Doppler frequency shift, and spectral linewidth of the scattered light provide measurements of density, velocity, and temperature, respectively ${ }^{1}$. Typical Rayleigh linewidths are on the order of 1-2 GHz; hence a very narrow linewidth laser ( $\sim 5 \mathrm{MHz}$ ) and an extremely high resolution filter are required to resolve the spectrum. These high resolution filters can be atomic or molecular absorption filters (filtered Rayleigh scattering ${ }^{2,3}$ ) or filters based on interference phenomena, such as the Fabry-Perot (FP) interferometer (interferometric Rayleigh scattering (IRS)). A FP interferometer ${ }^{4}$ consists of two parallel planar reflective plates or surfaces and is typically used in the imaging mode (constant spacing between reflective surfaces) for IRS, which is the approach taken in this work. When the FP interferometer has fixed plate spacing, it is often called an etalon. An etalon may be air-spaced or consist of a solid transparent optical material. When light is imaged through the etalon an interference pattern results which is a function of the spectrum of the light convolved with the instrument function of the FP. The FP instrument function is the well-known Airy function ${ }^{4}$.

In previous work where time-averaged measurements were acquired using a CCD detector to record the interference pattern ${ }^{5}$, the Rayleigh signal was transmitted to an air-spaced FP interferometer and detection equipment by an optical fiber. This allowed the FP interferometer to be remotely located from the harsh environments commonly encountered in test facilities. This is critical when using an air-spaced etalon that does not have a rigid fixed spacing since vibrations can change the plate spacing and corrupt the spectral measurements. Using an optical fiber to collect the Rayleigh scattered light provides point-wise or spatially-averaged measurements over a finite distance (on the order of $0.5 \mathrm{~mm}$ ) along a focused laser beam. Our research group at NASA Glenn Research Center has developed several fiber-coupled point-wise Rayleigh scattering measurement systems that are capable of acquiring data at high sampling rates using high quantum efficiency detectors ${ }^{6-8}$. There are many situations that would benefit from multiple-point measurements as well. Spatially-resolved measurements have been obtained by imaging scattered light from a laser line or sheet directly through a FP interferometer ${ }^{9-11}$. A direct imaging type of system requires that the interferometry equipment be located in or near the facility. An air-spaced Fabry-Perot is usually too delicate to operate in locations with high vibrations such as experienced in most NASA test facilities; therefore a solid etalon is typically used. A liquid crystal (LC) Fabry-Perot provides a robust 
interferometry system that is also tunable. Unlike temperature-tuned solid glass Fabry-Perot interferometers, the LC etalon can be tuned rapidly by adjusting the voltage applied to the liquid crystal interface.

Tuning the interferometer is often necessary to control the physical locations of the concentric interference fringes within the image. For spatially-resolved measurements, the measurement locations at the probe volume are dependent on the interference fringe positions in the detection plane. Therefore, tuning or scanning of the fringe positions is important to setting the measurement locations in the flow field. Also, maintaining stability of the fringe positions during data acquisition is critical to the velocity measurement accuracy ${ }^{12}$. In fiber-coupled Rayleigh measurement systems the imaged region of the fringe pattern is limited due to the small size of the fiber face that is imaged through the FP. In most cases only a fraction of an order of the interference pattern is imaged so that only the centermost fringe of the pattern is visible at the detector plane. It is possible for the centermost fringe to be a larger diameter than the imaged region such that the fringe is not visible on the detector. In this case it is necessary to have tuning capability to set the fringe radius such that the entire fringe falls within the imaged region. The fringe positions can be changed either by changing the laser frequency, which is not currently an option with the laser used in these experiments, or by adjusting the etalon spacing or the index of refraction of the medium in the etalon cavity. In the case of a solid etalon, both the spacing and refractive index can be changed by adjusting the temperature of the etalon; however standard fused silica etalons lack the fast tunability that is available with air-spaced interferometers equipped with piezoelectric transducers that change the mirror spacing in milliseconds. LC etalons contain a thin liquid crystal layer in conjunction with glass layers to form the solid etalon cavity. A custom designed LC etalon was built for this work in which the liquid crystal index of refraction can be varied from 1.5 to 1.75 by adjusting the voltage applied to the liquid crystal allowing the etalon properties to be rapidly changed with response times on the order of milliseconds, similar to tuning times for piezo-controlled air-spaced Fabry-Perot interferometers.

In previous characterization experiments Clem, et al. ${ }^{13}$ discovered that the LC etalon has a much lower free spectral range (FSR) than originally thought; the fringes are only about $2.5 \mathrm{GHz}$ apart. Typical FP's used in IRS have a FSR of about 8-10 GHz. The small FSR of the LC etalon resulted in some overlap of adjacent fringes since the full width at half maximum (FWHM) of the Rayleigh spectrum was about $2.2 \mathrm{GHz}$. This made it difficult to measure the Doppler shift and the Rayleigh linewidth very accurately in order to obtain velocity and temperature estimates. The experiment presented here was set up initially in a $90^{\circ}$ scattering configuration using a single-point, fiber-coupled imaging approach, rather than the direct-imaging configuration used previously ${ }^{13}$ to simplify the optical system and concentrate on the analysis of only the centermost fringe in the interference pattern in an effort to investigate the capabilities of the LC etalon. Data was acquired with the LC etalon as well as a fused silica (FS) solid etalon with similar spectral frequency resolution but with a higher FSR for comparison. Based on observations in the current and previous work, it was concluded that, due to non-optimal properties, the LC etalon is only useful in situations where the spectral linewidth is narrow compared to the FSR. This can be achieved by either lowering the flow temperature or reducing the magnitude of the interaction wave vector $(\mathbf{K})$. The temperature would have to be lowered to an experimentally unrealistic level to achieve the required linewidth reduction; therefore, the scattering vector was changed to $45^{\circ}$ relative to the incident laser beam to reduce the K-vector magnitude, and hence the linewidth, by a factor of 1.8. While the width of the Rayleigh spectrum was reduced it also took on a different shape due to an increase in the $y$-parameter, which is a non-dimensional parameter that defines the shape of the Rayleigh spectrum ${ }^{14,15}$. Side lobes or Brillouin scattering peaks were present on either side of the central Rayleigh peak. The separation between the Brillouin and Rayleigh peaks is related to the flow temperature. The resolution of both the FS and LC etalons was too low to resolve these narrow features; hence the $45^{\circ}$ scattering configuration was not capable of providing temperature estimates. However, the system did have the capability of measuring the overall radial shift of the spectrum to determine the velocity, and the signal strength to determine the gas density. Results from both the LC etalon and the FS etalon in the $90^{\circ}$ and $45^{\circ}$ scattering configurations applied in a 1-cm diameter free jet rig will be discussed.

\section{Rayleigh Scattering and Fabry-Perot Interferometry}

In molecular Rayleigh scattering an incident electric field interacts with an atom or a molecule inducing a dipole moment that oscillates and radiates at the frequency of the incident field. It is considered an elastic scattering process because the internal energy of the molecule is unchanged and the frequency of the light is changed only by the Doppler effect due to the thermal as well as the bulk motion of the molecules ${ }^{1}$. The frequency spectrum of the 


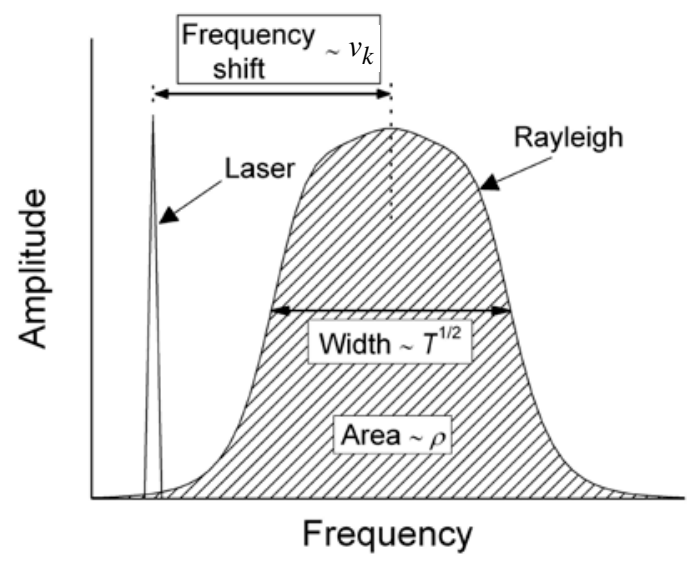

Fig. 1 Rayleigh scattering spectrum.

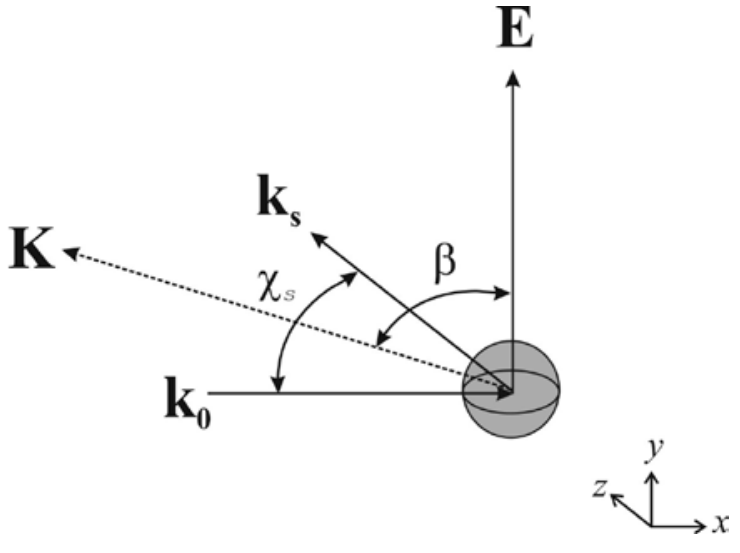

Fig. 2 Light scattering from a moving particle.

scattered light contains information about the gas density, bulk velocity, and temperature. Figure 1 shows a Rayleigh scattering spectrum containing the narrow laser line and a typical Rayleigh spectral peak for $90^{\circ}$ scattering relative to the incident laser beam to illustrate how the flow property measurements are obtained from the spectral information. If the gas composition is fixed, the total intensity of the Rayleigh scattered light is directly proportional to the gas density $\rho$. The frequency shift between the laser peak and the Rayleigh peak is proportional to the bulk flow velocity $v_{k}$. The width of the spectrum is related to the gas temperature $T$. The measured velocity component, $v_{k}$, is in the same direction as the interaction wave vector $\mathbf{K}$, which is the bisector of the incident and scattered light wave vectors, $\mathbf{k}_{\mathbf{0}}$ and $\mathbf{k}_{\mathbf{s}}$, respectively, as shown in Fig. 2 . The interaction wave vector and its magnitude $K$ are given by:

$$
\begin{gathered}
\mathbf{K}=\mathbf{k}_{\mathbf{s}}-\mathbf{k}_{\mathbf{0}} \\
K=|\mathbf{K}|=\frac{4 \pi}{\lambda}\left[\sin \frac{\chi_{s}}{2}\right]
\end{gathered}
$$

The geometry of the optical arrangement in a particular experiment determines the component $v_{k}$ of the velocity vector $\mathbf{v}$ that is measured:

$$
v_{k}=\frac{\mathbf{K} \cdot \mathbf{v}}{K}
$$

Experiments are typically arranged such that the electric field vector $\mathbf{E}$ is perpendicular to the scattering plane defined by the incident and scattered light wave vectors $\left(\beta=90^{\circ}\right.$, 's'-type or vertical polarization (Fig. 2)). In this configuration the collected scattering intensity is independent of the scattering angle and maximized.

The shape of the spectrum is dependent on gas pressure $p$ and temperature $T$, and the scattering angle $\chi_{\mathrm{s}}{ }^{14}$. A non-dimensional parameter $y$, is used to establish spectral shape regimes ${ }^{15}$ :

$$
y=\frac{p}{\eta K a}
$$

where

$$
a=\sqrt{\frac{2 \kappa T}{m}}
$$




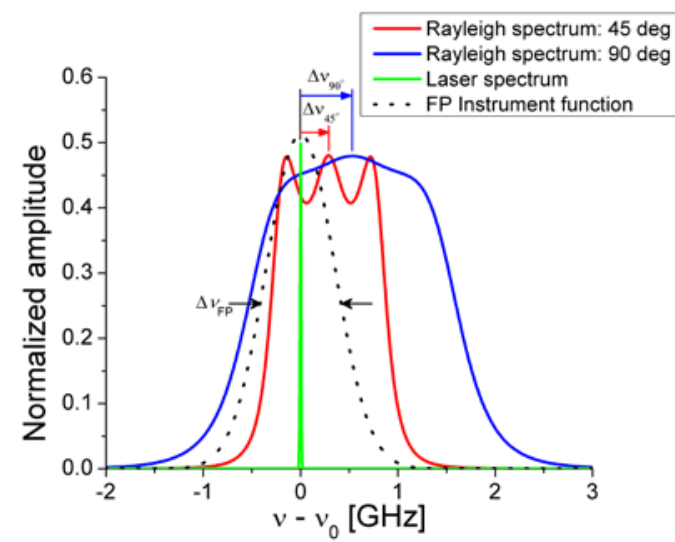

Fig. 3 Rayleigh spectra for Mach 0.6 flow $(T=278 \mathrm{~K}, v=200 \mathrm{~m} / \mathrm{s})$ in $45^{\circ}$ and $90^{\circ}$ scattering configurations, the laser spectrum, and the approximate Fabry-Perot instrument function for an etalon with $90 \%$ reflectivity.

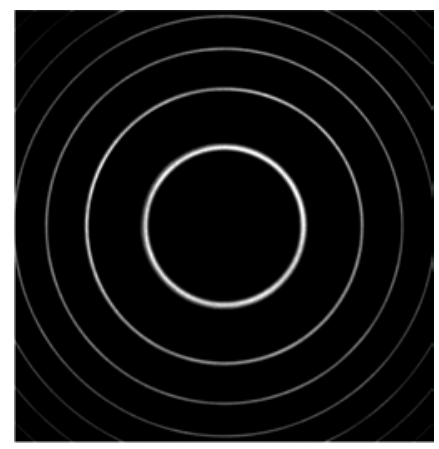

Fig. 5 Concentric ring inter-ference pattern from a planar singlefrequency light source imaged through a Fabry-Perot etalon.

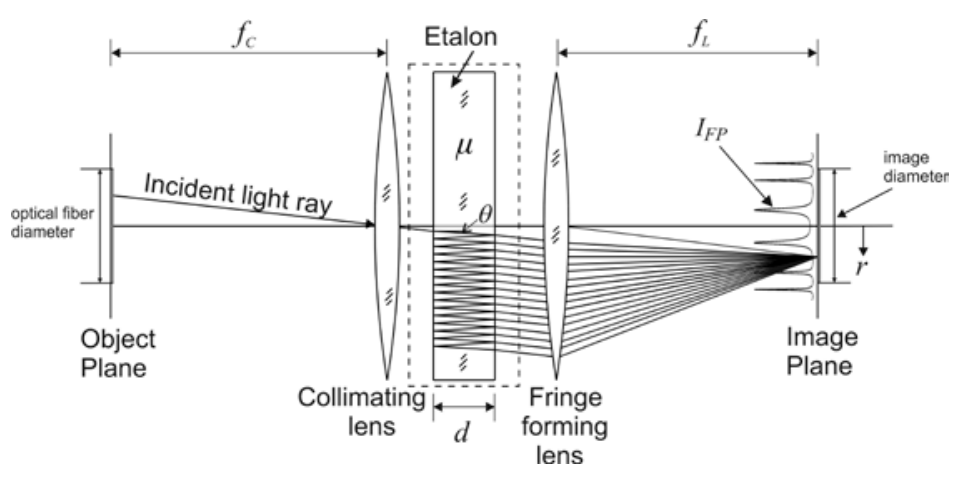

Fig. 4 Basic optical arrangement for spectrally-analyzing a light source using a solid Fabry-Perot etalon.

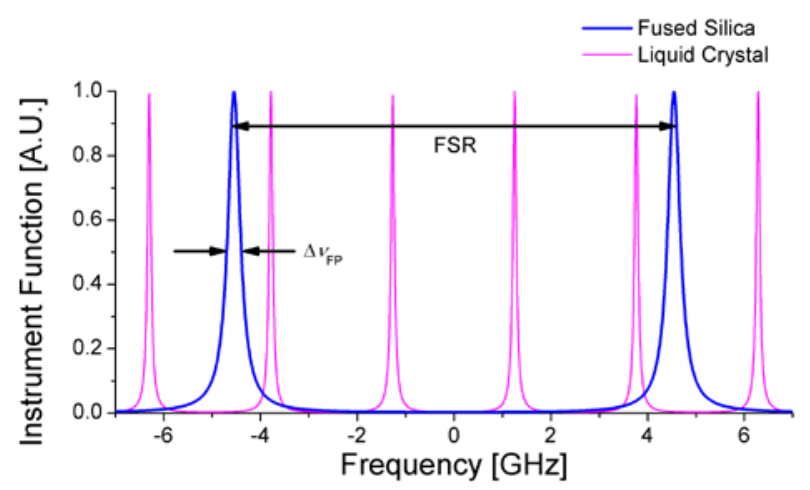

Fig. 6 Fabry-Perot Instrument functions for the fused silica (blue line) and the liquid crystal (magenta line) etalons. The FSR and resolution $\Delta v_{F P}$ are indicated for the fused silica etalon to illustrate how these are defined.

When $y$ is much less than 1 , the spectrum is accurately modeled by a Gaussian function. For values close to 1 the spectrum has a shape like that shown in Fig. 1 where the spectrum appears to have shoulders. As $y$ increases further the spectrum starts to develop side lobes known as Brillouin peaks. In the experiments presented in this work the $y$ parameter is about 0.86 and 1.6 for the $90^{\circ}$ and $45^{\circ}$ scattering configurations, respectively. The shapes of these two Rayleigh spectra are shown in Fig. 3. A Fabry-Perot interferometer or etalon is commonly used to resolve the Rayleigh spectrum. A detailed discussion of the theory on FP interferometry as applied to this type of Rayleigh scattering experiment can be found in References 16 and 17. A common arrangement for spectrally-analyzing Rayleigh scattered light using a planar Fabry-Perot interferometer is shown in Fig. 4. A typical interference fringe pattern that results at the image plane when a planar, uniform, single-frequency light source at the object plane is imaged through a FP etalon is shown in Fig. 5. The resulting interference pattern is the spectrum of the light convolved with the instrument function of the Fabry-Perot, which is the Airy function. The instrument functions as a function of frequency for the two etalons used in this work are illustrated in Fig. 6. In the frequency domain (Fig. 6) the fringes are equally spaced, however in the spatial domain (Fig. 5) the fringes are unequally spaced since the fringe order is proportional to $r^{2}$. The two key parameters that must be considered when selecting an etalon for use in Rayleigh scattering experiments are the FSR and the resolution, which are illustrated in Fig. 6. The FSR is the 
frequency change between consecutive fringe orders and it is set by the gap thickness between the reflective surfaces, $d$, and the refractive index of the medium in the gap, $\mu$ :

$$
\mathrm{FSR}=\frac{c}{2 \mu d}
$$

The resolution or FWHM of the instrument function, $\Delta \nu_{\mathrm{FP}}$, is a function of the FSR and the finesse, $\mathcal{F}$ :

$$
\Delta v_{\mathrm{FP}}=\frac{\mathrm{FSR}}{\mathcal{F}}
$$

where the finesse is dominantly a function of reflectivity:

$$
\mathcal{F} \approx \frac{\pi}{2} \frac{1}{\sin ^{-1}\left(\frac{1-R}{2 \sqrt{R}}\right)}
$$

The actual finesse is always lower than the ideal reflective finesse due to imperfect surface and coating quality. The effective finesse was measured to be about 14 for the FS etalon and 8 for the LC etalon, whereas the reflective finesse was about 30 for both etalons. The FSR of the LC etalon was about four times lower than that of the FS etalon as shown in Fig. 6 by the reduced spacing between fringes for the LC etalon. In general, an etalon must be chosen such that $\Delta v_{\mathrm{FP}}$ is less than the narrowest feature in the Rayleigh spectrum and the FSR is at least twice the linewidth of the spectrum to be measured.

In addition to the Rayleigh spectra, the laser spectrum and the Fabry-Perot instrument function used to resolve the spectra are also shown in Fig. 3 to give perspective about the relative widths of the various features. The FWHM of the $45^{\circ}$ scattering Rayleigh spectrum is about $1.2 \mathrm{GHz}$ whereas the $90^{\circ}$ scattering spectrum is about $2.2 \mathrm{GHz}$. The laser linewidth is approximately $5 \mathrm{MHz}$ and the FWHM of the Fabry-Perot instrument function $\left(\Delta \nu_{\mathrm{FP}}\right)$ is about 650 $\mathrm{MHz}$ for the FS etalon and $300 \mathrm{MHz}$ for the LC etalon (not shown in Fig. 3). The frequency shift between the laser line and the Rayleigh spectra is a function of the flow velocity and the magnitude of the K-vector:

$$
v-v_{0}=\frac{v_{k} K}{2 \pi}
$$

The frequency shift is greater for the $90^{\circ}$ scattering case shown in Fig. 3 since the magnitude of the K-vector is higher. The frequency shift for the $90^{\circ}$ scattering case $\left(\Delta v_{90^{\circ}}\right)$ is about $530 \mathrm{MHz}$ compared to the frequency shift for the $45^{\circ}$ scattering case $\left(\Delta v_{45^{\circ}}\right)$ which is about $290 \mathrm{MHz}$ based on a $200 \mathrm{~m} / \mathrm{s}$ flow. To evaluate the flow temperature from the $45^{\circ}$ spectrum the three peaks of the spectrum need to be accurately resolved. The resolution of the current etalons is insufficient to resolve the narrow Brillouin peaks which are on the order of $170 \mathrm{MHz}$ wide and separated by about $430 \mathrm{MHz}$ from the central Rayleigh peak. A Fabry-Perot interferometer with higher reflectivity and hence higher finesse would be needed to resolve these features and obtain temperature measurements in the $45^{\circ}$ scattering optical system configuration. Lowering the FSR to achieve higher resolution is not really an option in this case because the reduced FSR would not allow for sufficient separation between adjacent fringe orders in the interference pattern.

When spectrally broadened Rayleigh scattered light is imaged through the Fabry-Perot etalon the linewidth of the interference fringes provides a measure of temperature in the $90^{\circ}$ scattering configuration. The frequency shift of the light that is associated with the bulk flow velocity results in a change in the fringe radii. In fiber-coupled Rayleigh experiments the Rayleigh scattered light from a focused laser beam is collected into an optical fiber. The light exiting the optical fiber face (object plane in Fig. 4) is imaged through the FP interferometer onto a CCD detector (image plane in Fig. 4). Due to the finite diameter of the optical fiber only a single fringe order is typically imaged as shown in Fig. 7 where the narrow linewidth laser light exiting an optical fiber is imaged through a liquid crystal Fabry-Perot etalon and in Fig. 8 where thermally-broadened Rayleigh scattered light is imaged through the same system. The dashed line in both of these figures indicates the extent of the image of the fiber face. Any part of 


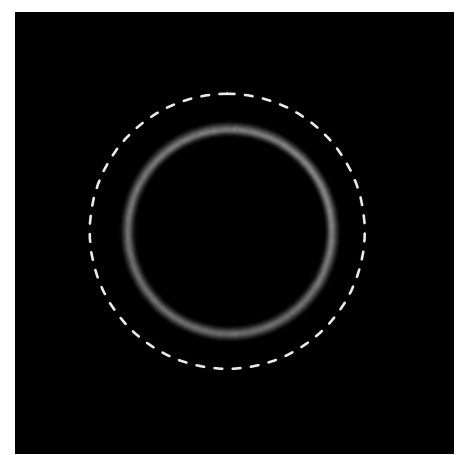

Fig. 7 Laser light exiting an optical fiber imaged through a liquid crystal Fabry-Perot etalon. The dashed line indicates the fiber image boundary.

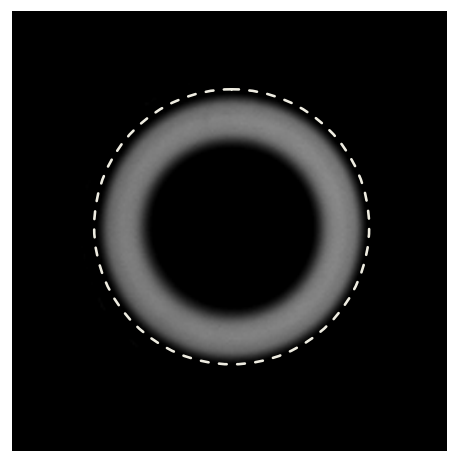

Fig. 8 Thermally-broadened Rayleigh scattered light exiting an optical fiber imaged through a liquid crystal Fabry-Perot etalon. The dashed line indicates the fiber image boundary.

the interference pattern that falls outside of that imaged region is not visible. The radius of the fringe in Fig. 8 is slightly larger than the radius of the fringe in Fig. 7 due to the Doppler frequency shift associated with a flow velocity of $190 \mathrm{~m} / \mathrm{s}$. The thermal motion of the molecules that are at $278 \mathrm{~K}$ causes the spectral broadening observed in Fig. 8 compared to the narrow linewidth of the fringe in Fig. 7.

In this experiment, Rayleigh scattered light from a defined probe volume is collected into a multimode optical fiber. The incident laser beam with power $P_{0}$ is focused to a diameter smaller than the imaged field size, such that the power collected is proportional to the length of the probe volume set by the field size of the collection optics. The Rayleigh scattered light integrated over the collection solid angle $\Omega$ from a probe volume of length $L$ expressed in terms of expected photoelectron counts is:

$$
\left\langle N_{R}\right\rangle=\int_{\Delta \Omega} \frac{\varepsilon P_{0} n L \lambda \Delta t \frac{\partial \sigma}{\partial \Omega} \sin ^{2} \beta}{h c} \partial \Omega
$$

where $\varepsilon$ is the overall system efficiency including detector quantum efficiency and other losses. With the interferometer in the optical path, the amount of energy, in terms of CCD grey level counts, collected on the $q^{\text {th }}$ pixel of the detector centered at position $\left(x_{q}, y_{q}\right)$ including light scattered from gas molecules (Rayleigh scattering), stray scattered light at the laser frequency, broadband background light and camera read noise is expressed as:

$$
\begin{gathered}
\left\langle N_{q}\right\rangle=\frac{A_{R}}{\varepsilon_{p}^{2}} \int_{y_{q}-\frac{\varepsilon_{p}}{2}}^{y_{q}+\frac{\varepsilon_{p}}{2}} \int_{x_{q}-\frac{\varepsilon_{p}}{2}}^{x_{q}+\frac{\varepsilon_{p}}{2}} \int_{-\infty}^{+\infty} S_{R}(v) I_{F P}(v, x, y) d v d x d y \\
+\frac{A_{f}}{\varepsilon_{p}^{2}} \int_{y_{q}-\frac{\varepsilon_{p}}{2}}^{y_{q}+\frac{\varepsilon_{p}}{2}} \int_{x_{q}-\frac{\varepsilon_{p}}{2}}^{x_{q}+\frac{\varepsilon_{p}}{2}} I_{F P}\left(v_{0}, x, y\right) d x d y+A_{b}
\end{gathered}
$$

where the amplitude of the Rayleigh scattered light is defined as:

$$
A_{R}=\left(\left\langle N_{R}\right\rangle \varepsilon_{p}^{2}\right) /\left(G \pi r_{\text {max }}^{2}\right)
$$

The second term in Eq. (11) is associated with scattered light at the laser frequency. The amplitude of broadband background light plus the camera read noise is represented by the constant $A_{b}$; this value is dominated by camera 
read noise since the level of broadband light in the room is minimized during testing. The Rayleigh spectrum $S_{R}$ is evaluated using the TENTI S6 kinetic theory mode ${ }^{14,15}$, which is a valid model over the full range of expected $y$ parameters. The imaged fringe patterns are analyzed by maximum likelihood estimation (MLE) ${ }^{18}$ analysis using the model function described in Eq. (11) to extract the density and velocity information, and also temperature information in the $90^{\circ}$ scattering experiments.

\section{Experiment}

\section{A. $90^{\circ}$ Rayleigh scattering measurements in a free jet flow}

Rayleigh scattering data was acquired in a flow exiting a free jet equipped with a $10 \mathrm{~mm}$ diameter convergent nozzle, at a $90^{\circ}$ scattering angle using both the FS and LC etalon systems separately. A top-view diagram of the $90^{\circ}$ Rayleigh scattering system is shown in Fig. 9. A Coherent Verdi $18 \mathrm{~W}$ continuous-wave $532 \mathrm{~nm}$ laser with a 2.25 $\mathrm{mm}$ diameter output beam and $5 \mathrm{MHz}$ linewidth provide the incident light for the system. A half-wave plate was used to rotate the polarization of the beam to orient the electric field vector perpendicular to the scattering plane at the probe volume in order to maximize the power of the scattered light in the direction of the collection optics. The laser beam was focused with a $175 \mathrm{~mm}$ focal length lens (L1) to a $1 / \mathrm{e}^{2}$ diameter of $50 \mu \mathrm{m}$ at the probe volume. The beam was oriented at a $45^{\circ}$ angle to the primary flow direction, while the scattered light was collected at a $90^{\circ}$ angle relative to the incident laser beam. The incident and scattering wave vectors were arranged such that the negative axial component of the jet velocity was measured as indicated by the red $\mathbf{K}$ vector in Fig. 9. A finite length of the laser beam was imaged by a pair of f/4 $200 \mathrm{~mm}$ focal length lenses (L2a and L3a) onto the face of a $0.55 \mathrm{~mm}$ diameter multimode optical fiber, shown in blue in Fig. 9. Since the lenses provided one-to-one imaging, the probe volume length was $0.55 \mathrm{~mm}$ along the laser propagation direction and the probe volume height, which was set by the focused beam waist, was $50 \mu \mathrm{m}$. The measurement probe volume was located on the jet centerline at an axial distance of $15 \mathrm{~mm}$ (1.5 jet diameters) from the nozzle exit. At the output of the laser a thin uncoated glass beamsplitter in the optical path sent $4 \%$ of the reference laser light to a diffuser where the light was collected and transmitted to the signal collection fiber (indicated in blue in Fig. 9) by another multimode fiber, referred to as the reference fiber (indicated in red in Fig. 9), and a small amount of laser light was coupled into the signal fiber by a 50 $\mathrm{mm}$ focal length lens (L4). A beam block mounted in a flipper apparatus was used to control whether or not reference laser signal was transmitted to the signal fiber. During data acquisition the beam block was initially out of the beam path to allow reference signal to be collected by the signal fiber. At this time, the collection optics (Lenses L2a and L3a) were blocked to allow only reference light to be coupled into the signal fiber. After acquiring the reference image, the beam block was placed in the beam path using the flipper apparatus to block the reference light from propagating to the signal fiber. The collection lens system was unblocked to allow Rayleigh signal to be transmitted through the collection system.

The signal fiber transmitted the collected signal to another optical table located in close proximity to the collection optics. The light exiting the fiber was collimated by a $60 \mathrm{~mm}$ focal length lens (L5) and was directed through the etalon. The FS and LC etalons were tested separately. The $25 \mathrm{~mm}$ diameter FS etalon had a refractive index of approximately 1.46 at room temperature and the $90 \%$ reflectivity coatings provided a reflective finesse of 30. The FS etalon was approximately $11.4 \mathrm{~mm}$ thick resulting in a FSR of $9 \mathrm{GHz}$. The $25 \mathrm{~mm}$ diameter LC etalon composed of fused silica layers and a very thin liquid crystal layer also had $90 \%$ reflectivity coatings giving a reflective finesse of 30. The effective index of refraction of the combined LC etalon materials at room temperature was approximately 1.49 and the total etalon thickness was about $40 \mathrm{~mm}$ giving a FSR of $2.5 \mathrm{GHz}$. Due to the ordinary and extraordinary states of the birefringent liquid crystal there were two sets of interference patterns that were transmitted simultaneously by the LC etalon. One set was static while the other was tunable with voltage. The transmission of these two states was polarization sensitive. Therefore, a linear polarizer was placed in front of the LC etalon to block the non-tunable state from passing through the etalon system. The light exiting the respective etalon system was focused by a Nikon $300 \mathrm{~mm}$ focal length lens (L6) onto the detector of a Princeton Instruments VersArray back-illuminated, scientific-grade CCD camera. The 512x512 imaging array had a 100\% fill factor, $24 \times 24 \mu \mathrm{m}$ pixels, 16-bit dynamic range, and thermoelectric cooling and low-noise electronics. Images were acquired with 1 second or 5 second exposure times, depending on the signal levels. The LC etalon had a lower signal throughput due to the linear polarizer in the beam path; therefore the longer exposure time was used for those experiments. 


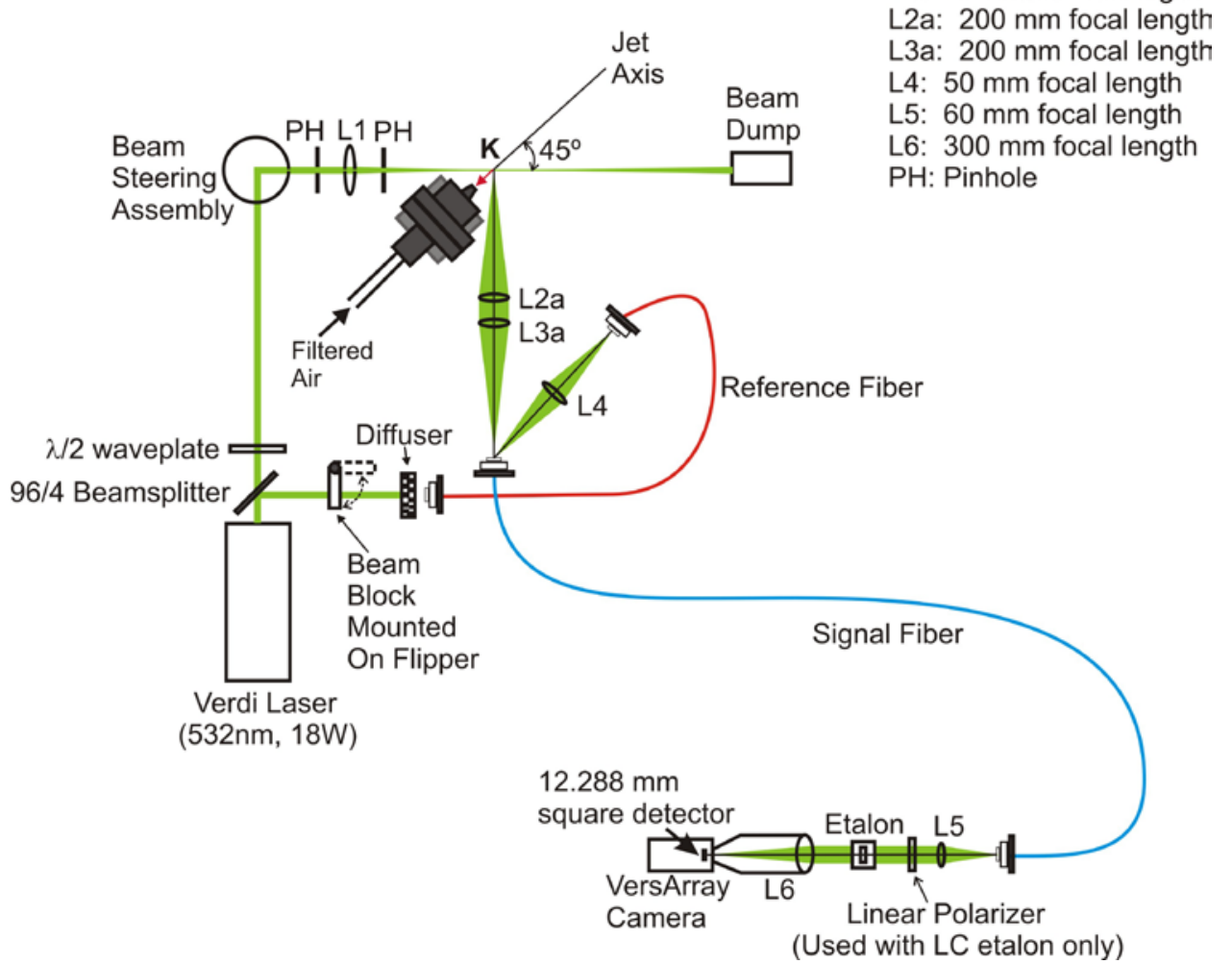
Fig. 9 Experiment schematic for the $90^{\circ}$ scattering angle Rayleigh scattering
experiment in a free jet flow.

\section{B. $45^{\circ}$ Rayleigh scattering measurements in a free jet flow}

The $90^{\circ}$ Rayleigh scattering system described above was reconfigured to enable $45^{\circ}$ scattering. A top-view diagram of the $45^{\circ}$ Rayleigh scattering system is shown in Fig. 10. The Coherent Verdi $18 \mathrm{~W}$ continuous-wave 532 $\mathrm{nm}$ laser was again used to provide the incident light for the system. The vertically-polarized laser beam remained focused with the $175 \mathrm{~mm}$ focal length lens (L1) to a diameter of $50 \mu \mathrm{m}$ at the probe volume, however, the jet was reoriented such that the laser beam was at a $67.5^{\circ}$ angle to the primary flow direction and the scattered light was collected at a $45^{\circ}$ scattering angle relative to the incident laser beam. The incident and scattering wave vectors resulted in a measured velocity component along the primary flow direction as indicated by the red $\mathbf{K}$ vector in Fig. 10. A finite length of the laser beam was imaged by a pair of $f / 2100 \mathrm{~mm}$ focal length lenses (L2b and L3b) onto the face of the $0.55 \mathrm{~mm}$ multimode signal collection fiber, shown in blue in Fig. 10. Due to the one-to-one imaging provided by lenses L2b and L3b the probe volume length was $0.55 \mathrm{~mm}$ along the laser propagation direction and the probe volume width was $50 \mu \mathrm{m}$. Again, the measurement probe volume was located on the jet centerline at an axial distance of 1.5 jet diameters from the nozzle exit. The reference fiber (indicated in red in Fig. 10) transmitted reference laser light to the signal fiber and a small amount of laser light was coupled into the collection system by the $50 \mathrm{~mm}$ focal length lens (L4). The signal fiber transmitted the collected signal to the same etalon and detection system as described above. The same data acquisition process for the $90^{\circ}$ scattering was applied for the $45^{\circ}$ scattering system where a purely reference signal image was acquired first and a Rayleigh signal image was acquired about 15 seconds later. Again, the images acquired with the LC etalon required a 5 second exposure time while the FS etalon images could be acquired with a 1 second exposure time to provide ample signal for flow parameter estimation from the image data. 


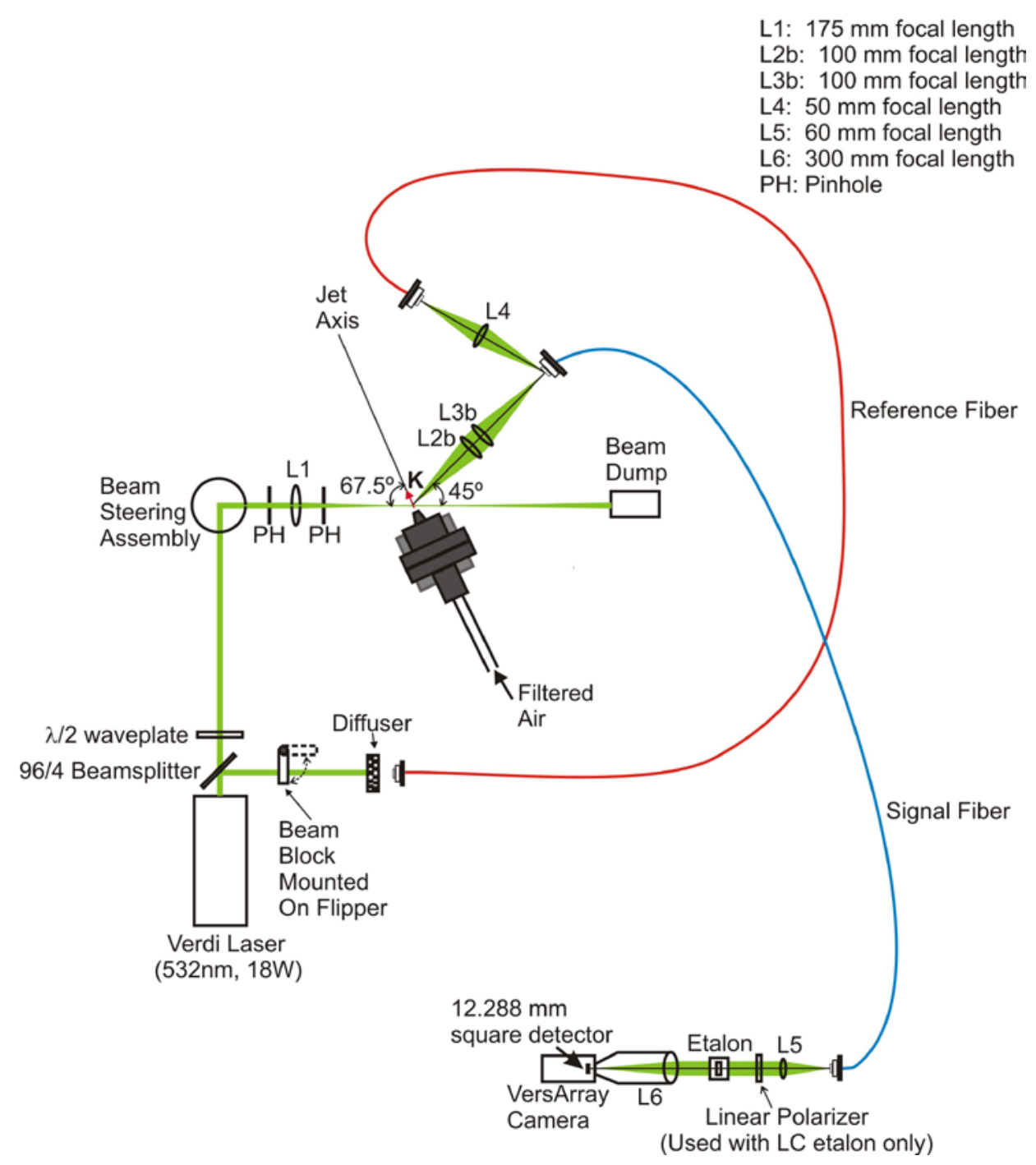

Fig. 10 Experiment schematic for the $45^{\circ}$ scattering angle Rayleigh scattering experiment in a free jet flow.

\section{Results}

Image data were acquired in the core of the jet flow at 1.5 jet diameters downstream of the nozzle exit. The jet was oriented relative to the optics in each scenario such that the Rayleigh diagnostic system was measuring the axial velocity component. A reference interference fringe image with incident laser light was acquired first and a fringe image of Rayleigh scattered light was acquired approximately 15 seconds later. The laser frequency drifted by a minimal amount during the time period between the two acquisitions. The laser frequency tended to drift at a fairly constant rate of about 1-2 MHz/s throughout the experiments and typically resulted in a 0.5-1.0 pixel change in reference fringe radius between the reference and Rayleigh images. This was taken into account in the image processing to minimize velocity errors due to laser frequency drift. Since the data were acquired in the jet core, isentropic flow equations were used to calculate theoretical velocity, density, and temperature estimates based on static pressure, total pressure, and total temperature measurements. These values were used to calibrate the optical system efficiency so that density estimates could be determined from the image data. The finesse value was determined from fitting both the reference and Rayleigh image data to the model function using MLE analysis and assuming theoretical flow properties. An average or "effective" finesse value for each etalon was determined and 
input into the model function for analysis of the data for flow measurement purposes. The effective finesse of the FS etalon was found to be about 14 and the effective finesse of the LC etalon was about 8.

Once the system was calibrated the MLE procedure was used to fit the model function to the reference and Rayleigh image data. The corrected reference fringe radius was determined from fitting the radius of the fringe in the reference image and applying a correction factor of 1.0 pixel to account for laser drift and was input into the model function to fit the Rayleigh fringe. The center position $\left(x_{0}, y_{0}\right)$ of the fringe image was also determined from the reference fringe and used in fitting the Rayleigh fringe. In the $45^{\circ}$ scattering experiments the stray laser light was not controlled as well as it was in $90^{\circ}$ scattering experiments so a small amount of stray light (10\% of total signal) had to be taken into account in the model function for those images. The Rayleigh fringe data were fit for density and velocity, and in the $90^{\circ}$ scattering FS etalon configuration the Rayleigh fringe data were also fit for temperature. Some typical data and model function fits for each experimental configuration are shown in Figs. 11-14.

Figure 11 shows the data (solid lines) and fits (symbols) for the FS etalon in the $90^{\circ}$ scattering configuration. Ideally the reference fringe should have been at a larger radius for these measurements because the Doppler shifted Rayleigh fringe (negative radial shift due to measurement of the negative axial velocity component) is very close to the center making it more difficult to accurately evaluate the fringe peak location and width for the velocity and temperature measurements, respectively. The FS etalon did not have tuning capabilities other than attempting to alter the ambient room temperature. The laser system also lacked the ability to control the output laser frequency. Therefore it was very difficult to get the starting fringe radius in the optimal location for the measurements. Figure 12 shows the data from the LC etalon in the $90^{\circ}$ configuration. Only the reference fringe has a model function fit shown since the Rayleigh image data was not able to be fit. This was due to the fact that the LC etalon has a FSR of only approximately $2.5 \mathrm{GHz}$ and the $2.2 \mathrm{GHz}$ wide Rayleigh spectral peaks overlap between consecutive fringe orders. The next higher and lower fringe orders are not visible in the narrow linewidth reference fringe data for the LC etalon, however once the very broad Rayleigh signal is present, the tails of the next higher and lower fringes spill into the imaged region and interfere with the fringe of interest. The resulting Rayleigh interference fringe pattern has no distinguishable peak to use for determining velocity or temperature measurements. These observations along with the results from a previous study of the LC etalon ${ }^{13}$ led to the decision to collect data at $45^{\circ}$ scattering to reduce the width of the Rayleigh spectrum.

Figures 13 and 14 show the data (solid lines) and fits to the model function (symbols) obtained from the FS and LC etalon experiments in the $45^{\circ}$ scattering configuration, respectively. The narrower spectral peaks can be measured with both the FS and LC etalons. The measured velocity component is in the same direction as the axial velocity so the radial shift between the reference fringe and Rayleigh fringe is positive. The central portion of the Rayleigh fringe image for the LC etalon was ignored in the MLE analysis as indicated by the lack of symbols in that region of the profile. The model function was not able to fit the central region accurately possibly due to contamination from the next lower order fringe and it caused the fringe peak location to be skewed to lower values. By fitting only the region around the peak the radius of the fringe was determined more accurately.

The velocity estimates based on MLE analysis of the fringe image data from the three experiment configurations that produced quality fringe images (FS and LC etalons at $45^{\circ}$ scattering and FS etalon at $90^{\circ}$ scattering) are shown in Fig. 15. The measured velocities in all three cases were within $8 \mathrm{~m} / \mathrm{s}$ of the theoretical values. The density estimates compared to the theoretical values for the three experiments are presented in Fig. 16 and the measurements all fall within $0.6 \%$ of the theoretical values. The only configuration that had the potential for estimating flow temperature was the FS etalon in the $90^{\circ}$ configuration. The temperature results from that experiment are shown in Fig. 17. The estimates are a bit low, by as much as $8 \mathrm{~K}$ in some cases. This is believed to be due to the fact that the starting reference fringe radius was too small such that the shifted fringe moved too far into the center of the interference pattern causing loss of information. The highest velocity, lowest temperature case is the one where the shifted fringe has moved the furthest into the center and this is the data point with the highest offset from the theoretical value. Alternatively, the lower velocity, higher temperature cases are the ones with the most accurate temperature results due to the larger radius of the shifted fringe.

A Cramer-Rao lower bound (CRLB) uncertainty analysis was performed to demonstrate how the lower bound on the uncertainty in the flow measurements changes with reference fringe radius. The details of this type of analysis have been reported previously in Ref. 8 and Ref. 12. The results of the analysis in terms of velocity and temperature 


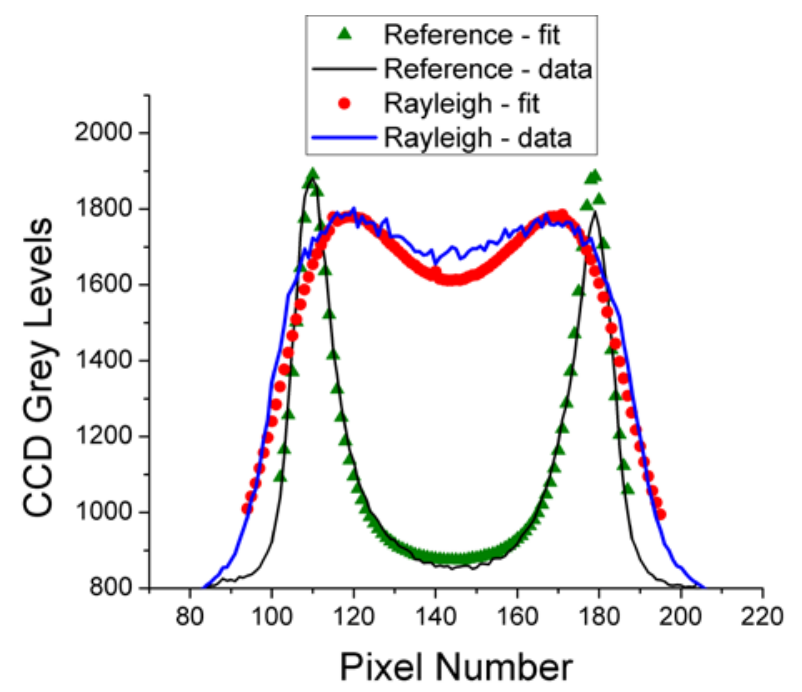

Fig. 11 Line profile through the reference and Rayleigh interference fringe data for the FS etalon at $90^{\circ}$ scattering along with the model function fit to each fringe. The velocity and temperature of the flow were $190 \mathrm{~m} / \mathrm{s}$ and $278 \mathrm{~K}$, respectively.

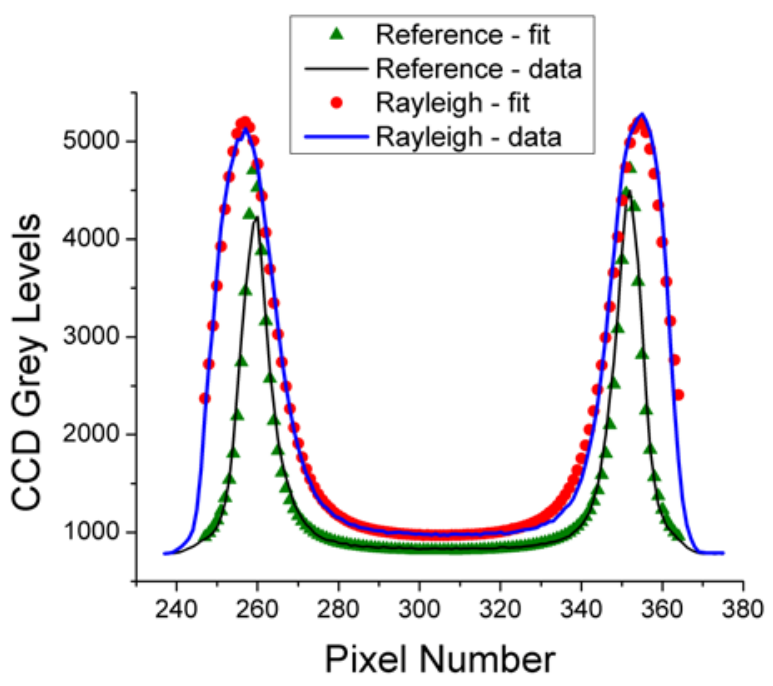

Fig. 13 Line profile through the reference and Rayleigh interference fringe data for the FS etalon at $45^{\circ}$ scattering along with the model function fit to each fringe. The velocity and temperature of the flow were $190 \mathrm{~m} / \mathrm{s}$ and 278 $\mathrm{K}$, respectively.

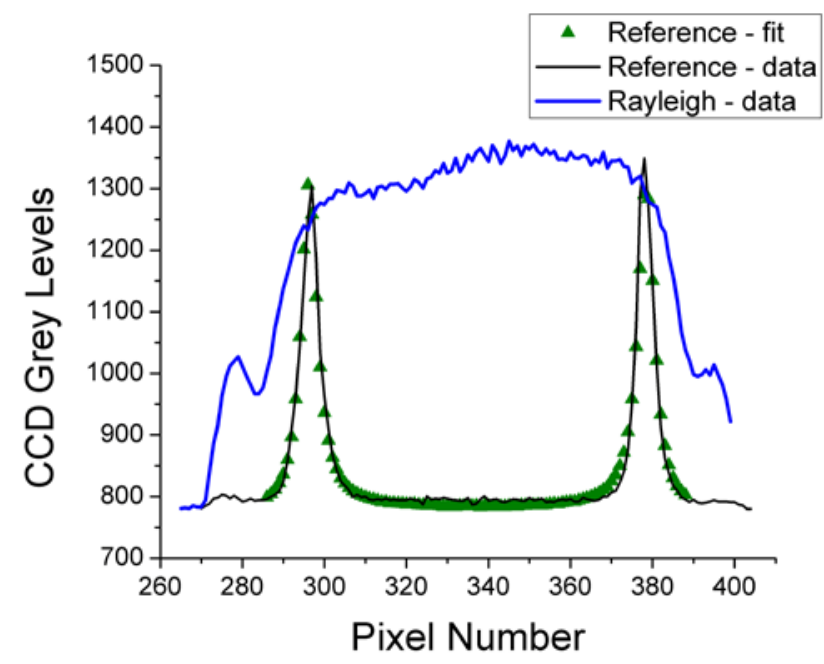

Fig. 12 Line profile through the reference and Rayleigh interference fringe data for the LC etalon at $90^{\circ}$ scattering. Only the model function fit to the reference image data is shown since it was not possible to fit the Rayleigh data. The velocity and temperature of the flow were $60 \mathrm{~m} / \mathrm{s}$ and $295 \mathrm{~K}$, respectively.

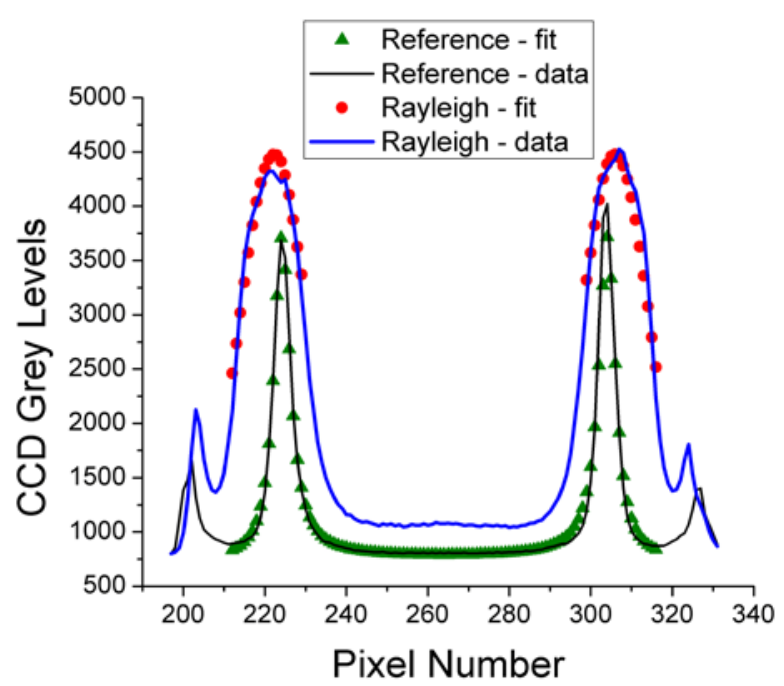

Fig. 14 Line profile through the reference and Rayleigh interference fringe data for the LC etalon at $45^{\circ}$ scattering along with the model function fit to each fringe. The velocity and temperature of the flow were $190 \mathrm{~m} / \mathrm{s}$ and $278 \mathrm{~K}$, respectively. The central portion of the fringe had to be ignored to get a good peak estimate since the start of another fringe coming into the center tended to skew the radius toward the center. 
measurement uncertainty as a function of reference fringe radius are shown in Fig. 18 for a flow with a velocity of $190 \mathrm{~m} / \mathrm{s}$ and a temperature of $278 \mathrm{~K}$ in the FS etalon $90^{\circ}$ scattering experiment configuration. Keep in mind that this is the lower bound on the uncertainty so the measurement uncertainty could be higher, but this is the lower limit of what can be achieved. The density uncertainty is not shown because it is linearly related to signal strength and the total signal strength in this CRLB exercise was constant. The CRLB analysis shows that both temperature and velocity uncertainty decrease as the reference fringe radius increases until the uncertainties reach a minimum at a radius of $1.1 \mathrm{~mm}$. Beyond a radius of $1.1 \mathrm{~mm}$ the uncertainty starts to increase again as the fringe width starts to go beyond the extent of the fiber image and information about the peak is lost. The FS etalon measurements at $90^{\circ}$ scattering were acquired with a fringe radius of about $0.8 \mathrm{~mm}$ whereas the optimal fringe radius was $1.1 \mathrm{~mm}$. The strong dependence of the Rayleigh measurement uncertainty on the starting reference fringe radius reiterates the need for a tunable fringe system, either by tuning the etalon properties or the laser frequency. This is especially true in fiber-coupled experiments where the imaged region of the interference pattern is limited and the measurements rely on the lowest order fringe being strategically located.

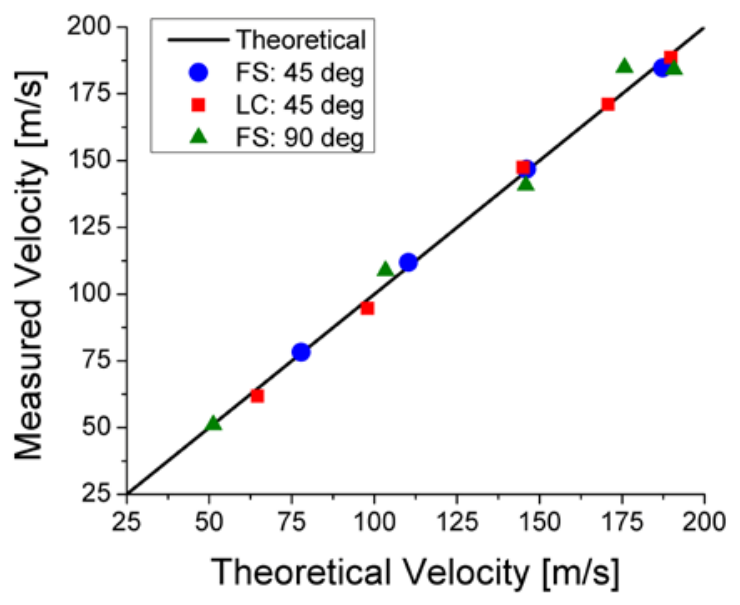

Fig. 15 Velocity measurement results compared to theoretical values for three experiment configurations: FS etalon in both $45^{\circ}$ and $90^{\circ}$ scattering configurations and the LC etalon in the $45^{\circ}$ scattering configuration.

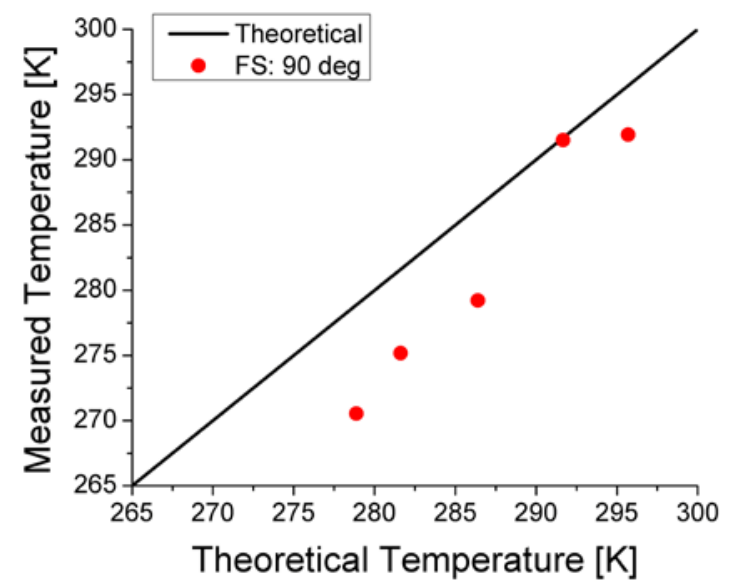

Fig. 17 Temperature measurement results compared to theoretical values for FS etalon in the $90^{\circ}$ scattering configuration.

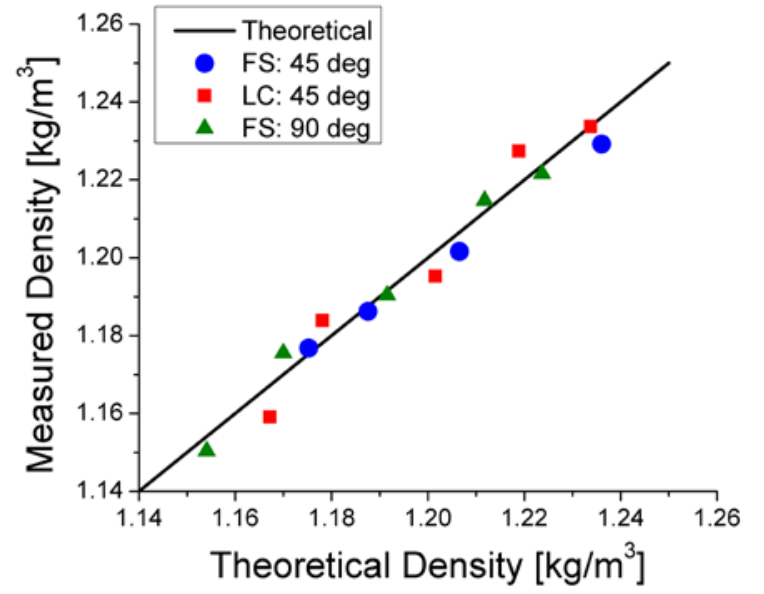

Fig. 16 Density measurement results compared to theoretical values for three experiment configurations: FS etalon in both $45^{\circ}$ and $90^{\circ}$ scattering configurations and the LC etalon in the $45^{\circ}$ scattering configuration.

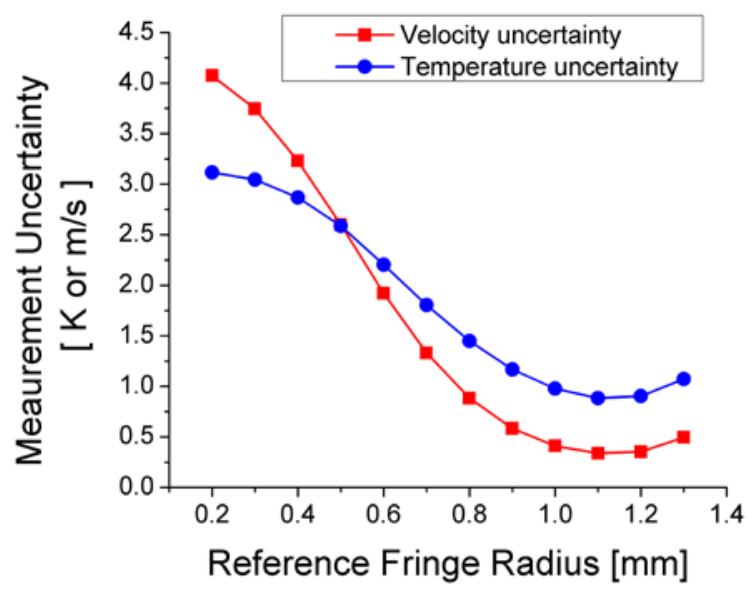

Fig. 18 Cramer-Rao Lower Bound uncertainty analysis results showing how the starting reference fringe radius affects both velocity and temperature measurement uncertainty in a $90^{\circ}$ scattering configuration with a flow velocity of $190 \mathrm{~m} / \mathrm{s}$ and gas temperature of $278 \mathrm{~K}$ for the FS etalon. 


\section{Conclusions, Recommendations and Future Work}

Various Rayleigh scattering configurations and etalon types were used to measure the axial velocity, density, and temperature of a 1-cm diameter nozzle free jet flow. Experiments were performed using a tunable liquid crystal etalon that had layers of fused silica in combination with a thin layer of liquid crystal to provide tuning capability via controlling the voltage applied to the liquid crystal interface. Experiments were also performed with a standard fused silica etalon that did not have any tuning capabilities. These two etalons were tested in $90^{\circ}$ and $45^{\circ}$ scattering configurations to establish their usefulness in Rayleigh scattering experiments. The Rayleigh spectral linewidth at $90^{\circ}$ scattering was expected to be problematic in the liquid crystal etalon configurations due to limited free spectral range; hence the $45^{\circ}$ scattering configuration was used to provide a narrower Rayleigh spectrum.

The fused silica etalon was able to provide velocity and density measurements that were within $8 \mathrm{~m} / \mathrm{s}$ and $0.6 \%$ of the theoretical velocity and density, respectively, in both scattering configurations. Temperature measurements were presented for the $90^{\circ}$ scattering fused silica etalon experiments; however temperature measurements were not possible in the $45^{\circ}$ scattering experiment since the resolution of the etalon was insufficient to resolve the Brillouin peaks that were present in the Rayleigh spectrum at that scattering angle. The temperature and velocity measurements could have been improved if the etalon had tuning capabilities to set the starting reference fringe radius at the optimal location, as determined by a Cramer-Rao lower bound analysis, to minimize measurement uncertainty.

The liquid crystal etalon was only able to provide velocity and density measurements in the $45^{\circ}$ scattering configuration. The measurement errors were similar to the fused silica measurements. Temperature measurements were not possible at $45^{\circ}$ scattering for the same reason as the fused silica etalon; the resolution of the etalon instrument function was insufficient to resolve the Brillouin peaks. Flow measurements were not possible at all with the liquid crystal etalon in the $90^{\circ}$ scattering configuration since the free spectral range of this etalon was only 2.5 $\mathrm{GHz}$ which resulted in signal overlap between adjacent peaks in the interference pattern since the Rayleigh spectral peak is about $2.2 \mathrm{GHz}$ wide in the $90^{\circ}$ scattering configuration.

In general, when selecting an etalon for use in Rayleigh scattering or any other spectroscopic diagnostic, it is important to chose an etalon with frequency resolution better than the narrowest feature in the spectrum to be measured and with a free spectral range at least twice the linewidth of the spectrum of interest. Additionally, if the imaged region of the interference pattern is limited to less than one free spectral range it is advantageous to have the capability to tune either the etalon or the incident laser frequency to optimize the fringe positions such that the uncertainty in the measurements is minimized.

In future work, we plan to implement some level of temperature control for the fused silica etalon since it has better optical properties for Rayleigh scattering work in terms of free spectral range and it would be useful to have some way to control the starting fringe radius, even if the control has a slow response time and is not as accurate as the liquid crystal etalon control. We may also investigate methods of tuning the laser frequency by adjusting the temperature of the vanadate crystal in the laser head, which would give an alternate method of setting the starting reference fringe radius. We also plan to obtain a fused silica etalon with higher reflectivity, and hence higher resolution, to investigate temperature measurements in experimental systems where the Rayleigh spectrum contains Brillouin peaks. Using the width of the Rayleigh spectrum as a measure of temperature has always been difficult since the width is affected by other factors, such as turbulence broadening, in addition to thermal broadening. Using the Brillouin peak locations as a measure of temperature may be a more accurate way of measuring temperature in Rayleigh scattering experiments.

\section{Acknowledgments}

The authors would like to thank Professor Tenti of the University of Waterloo for provide the TENTI S6 numerical code for calculating the Rayleigh spectrum. This work is supported by the Hypersonics discipline under NASA's Fundamental Aeronautics Program. 


\section{References}

${ }^{1}$ Miles, R. B., Lempert, W. R., and Forkey, J. N., “Laser Rayleigh Scattering,” Meas. Sci. Technol., Vol. 12, No. 5, 2001, pp. R33-R51.

${ }^{2}$ Forkey, J. N., Lempert, W. R., and Miles, R. B., “Accuracy Limits for Planar Measurements of Flow Field Velocity, Temperature, and Pressure Using Filtered Rayleigh Scattering,” Exp. Fluids, Vol. 24, No. 2, 1998, pp. 151-162.

${ }^{3}$ Boguszko, M., and Elliott, G. S., "On the Use of Filtered Rayleigh Scattering for Measurements in Compressible Flows and Thermal Fields,” Exp. Fluids, Vol. 38, No. 1, 2005, pp. 33-49.

${ }^{4}$ Vaughan, J. M., The Fabry Perot Interferometer, History, Theory, Practice, and Applications, Adam Hilger, Philadelphia, 1989, pp. 89-134.

${ }^{5}$ Mielke, A. F., Seasholtz, R. G., Elam, K.A., and Panda, J., “Time-average Measurement of Velocity, Density, Temperature, and Turbulence Velocity Fluctuations Using Rayleigh and Mie Scattering,” Experiments in Fluids, Vol. 39, No. 2, 2005, 441454.

${ }^{6}$ Panda, J., and Seasholtz, R. G., "Velocity and Temperature Measurement in Supersonic Free Jets Using Spectrally Resolved Rayleigh Scattering,” AIAA-99-0296, 1999.

${ }^{7}$ Seasholtz, R. G., Panda, J., and Elam, K. A., "Rayleigh Scattering Diagnostic for Measurement of Velocity and Density Fluctuation Spectra," AIAA-2002-0827, 2002.

${ }^{8}$ Mielke, A. F., Elam, K. A., and Sung, C. J., "Multiproperty Measurements at High Sampling Rates Using Rayleigh Scattering,” AIAA Journal, Vol. 47, No. 4, 2009, 850-862.

${ }^{9}$ Seasholtz, R. G., Buggele, A. E., and Reeder, M. F., "Flow Measurements Based on Rayleigh Scattering and Fabry-Perot Interferometer," Optics and Lasers in Engineering, Vol. 27, No. 6, 1997, pp. 543-570.

${ }^{10}$ Bivolaru, D., Danehy, P. M., Gaffney, R. L., and Cutler, A. D., "Direct-View Multi-Point Two-Component Interferometric Rayleigh Scattering Velocimeter,” AIAA-2008-236, 2008.

${ }^{11}$ Lock, J. A., Seasholtz, R. G., and John, W. T., "Rayleigh-Brillouin Scattering to Determine One-Dimensional Temperature and Number Density Profiles of a Gas Flow Field,” Applied Optics, Vol. 31, No. 15, 1992, pp. 2839-2848.

${ }^{12}$ Mielke, A. F., "Development of a Molecular Rayleigh Scattering Diagnostic for Simultaneous Time-Resolved Measurement of Temperature, Velocity, and Density," Ph.D. Dissertation, Department of Mechanical and Aerospace Engineering, Case Western Reserve Univ., Cleveland, OH, 2008, pp. 170-176.

${ }^{13}$ Clem, M. M., Mielke-Fagan, A. F., Elam, K. A., "Study of Fabry-Perot Etalon Stability and Tuning for Spectroscopic Rayleigh Scattering,” AIAA-2010-855, 2010.

${ }^{14}$ Tenti, G., Boley, C. D., and Desai, R. C., "On the Kinetic Model Description of Rayleigh-Brillouin Scattering from Molecular Gases,” Canadian Journal of Physics, Vol. 52, No. 4, 1974, pp. 285-290.

${ }^{15}$ Boley, C. D., Desai, R. C., and Tenti, G., "Kinetic models and Brillouin scattering in a molecular gas," Canadian Journal of Physics, Vol. 50, No. 18, 1972, pp. 2158-2173.

${ }^{16}$ Mielke-Fagan, A. F., Clem, M. M., Elam, K. A., and Hirt, S. M., "Progress on a Rayleigh Scattering Mass Flux Measurement Technique,” AIAA-2010-856, 2010.

${ }^{17}$ Mielke-Fagan, A. F., Elam, K. A., and Clem, M. M., "Multiple-Point Mass Flux Measurement System Using Rayleigh Scattering,” AIAA-2009-528, 2009.

${ }^{18}$ Edwards, R. V., Processing Random Data: Statistics for Engineers and Scientists, World Scientific, New Jersey, 2006, pp.91-97. 